\title{
ONE POT SOLVOTHERMAL SYNTHESIS OF ORGANIC ACID COATED MAGNETIC IRON OXIDE NANOPARTICLES
}

\author{
VERÓNICA PAREDES-GARCÍA *1,2, NÉSTOR TOLEDO' ${ }^{2}$ JULIANO DENARDIN ${ }^{2,3}$, DIEGO VENEGAS-YAZIGI ${ }^{2,4}$, \\ CARLOS CRUZZ, EVGENIA SPODINE ${ }^{2,5}$, ZHIPING LUO
}

\author{
${ }^{1}$ Universidad Andres Bello, Departamento de Ciencias Quimicas, Chile. \\ ${ }^{2}$ CEDENNA, Chile \\ ${ }^{3}$ Facultad de Ciencia, Universidad de Santiago de Chile, Chile \\ ${ }^{4}$ Facultad de Química y Biología, Universidad de Santiago de Chile, Chile \\ ${ }^{5}$ Facultad de Ciencias Químicas y Farmacéuticas, Universidad de Chile, Chile \\ ${ }^{6}$ Microscopy and Imaging Center and Materials Science and Engineering Program, Texas A\&M University, College Station, TX 77843, USA \\ 7 Department of Chemistry and Physics, Fayetteville State University, Fayetteville, NC 28301, USA
}

(Received: July 19, 2013 - Accepted: November 19, 2013)

\begin{abstract}
In this work we present the synthesis and characterization of iron oxide nanoparticles (IONPs), which were structurally and magnetically characterized. The use of iron salts and an organic acid (l-serine or ascorbic acid) as precursors under solvothermal conditions yielded these coated IONPs. The powder X-ray diffraction pattern of FeO-1 and FeO-2 is consistent with hematite $\left(\alpha-\mathrm{Fe}_{2} \mathrm{O}_{3}\right)$ and hematite-maghemite $\left(\left(\alpha-\mathrm{Fe}_{2} \mathrm{O}_{3} / \gamma-\mathrm{Fe}_{2} \mathrm{O}_{3}\right)\right.$ respectively. The TEM analysis permits to estimate an average size of $10 \mathrm{~nm}$ for the $\mathrm{FeO}-1$ sample. The magnetic characterization of the samples through the $M(H)$ plots showed a very low coercivity value for both samples, being 53 Oe for FeO-1 and 10 Oe for FeO-2, indicating the very weak ferromagnetic character of the synthesized iron oxide species. Even though both organic acids under solvothermal conditions permit to obtain coated IONPs in one pot reaction, l-serine produces a more narrow-size distribution.
\end{abstract}

Keywords: Iron oxide, nanoparticles, hematite, maghemite, solvothermal synthesis, magnetism

\section{INTRODUCTION}

Nanoparticles (NPs), correspond to organic or inorganic submicron moieties that usually have novel properties compared with the bulk material [1]. Within the inorganic nanoparticles it is possible to find the magnetic NPs, which are characterized by the presence of unusual magnetic properties. High coercivity, low Curie temperature, high magnetic susceptibility or superparamagnetism is characteristics of the magnetic NPs [2].

The interest to synthesize and study the magnetic NPs is due to a wide variety of applications in fields such as magnetic fluids, data storage, catalysis, biology and medicine, among others [3]. For example, the use of these type of nanoparticles in magnetic ferrofluids and data storage has allowed innumerable commercial applications $[2,4]$. Currently, the magnetic nanoparticles are being studied primarily due to the potential applications in biology and biomedicine. Targeted drug delivery, magnetic fluid hyperthermia (MFH), magnetic resonance imaging (MRI), magnetic bioseparations are some examples, where the magnetic NPs are being applied [5]. However, the choice of the magnetic nonmaterial depends on which type of application is desired. For instance, magnetically hard NPs are desirable for data storage, while all biological and biomedical applications require NPs which are biocompatible, non-toxic and chemically stable $[5 \mathrm{a}, \mathrm{b}-6]$.

Iron oxide nanoparticles (IONPs) belong to a special class of magnetic nanoparticles, which have been widely studied due to their scientific and industrial applications, which can range from data storage to MRI. There are different types of iron oxides, such as wustite (FeO), mixed iron (II, III) oxide $\left(\mathrm{Fe}_{3} \mathrm{O}_{4}\right.$, magnetite), and iron (III) oxides, including alpha phase $\left(\left(\alpha-\mathrm{Fe}_{2} \mathrm{O}_{3}\right.\right.$, hematite), beta phase $\left(\beta-\mathrm{Fe}_{2} \mathrm{O}_{3}\right)$, gamma phase $\left(\gamma-\mathrm{Fe}_{2} \mathrm{O}_{3}\right.$, maghemite), and epsilon phase $\left(\varepsilon-\mathrm{Fe}_{2} \mathrm{O}_{3}\right)[7]$.

Many synthetic methods have been used to obtain magnetic iron oxide nanoparticles. These include organic solvent heating method, polyol method, and co-precipitation method $[2,8]$. Furthermore, several biological compounds have been used as surface coatings to prevent the aggregation of the NPs, for control size and biocompatibility [8]. However, the biomedical and bioengineering applications of the superparamagnetic iron oxide nanoparticles (SPIONs) are limited by the magnetic properties and size of these NPs. SPIONs having high magnetization values, size smaller than $100 \mathrm{~nm}$, and an overall narrow particle size distribution, are very attractive due to the fact that they present uniform physical and chemical properties [9]. Beyond the magnetic applications, magnetite and hematite have also been used as catalysts for a number of industrially important reactions such as the Haber process (synthesis of $\mathrm{NH}_{3}$ ), desulfurization of natural gas, dehydrogenation of ethyl benzene to styrene, the Fisher-Tropsch synthesis for hydrocarbons, oxidation of alcohols, and others [3e-i]. Besides, the three forms of magnetic iron oxide are used in synthetic pigments in paints, ceramics, and porcelain. However, the utility and therefore the applications of the iron oxides depend on the particle size and shape, size distribution, surface chemistry, degree of structural defects or impurities present in the particles, which can be related to the used synthetic procedure [3j-1].

Because of these extensive technological and industrial applications of the different iron oxide compounds, finding new synthetic routes to obtain this class of oxides is very motivating. In this work, we inform the synthesis and characterization of two types of iron oxides magnetic nanoparticles ( $\mathrm{FeO}-1$ and $\mathrm{FeO}-2$ ), which were obtained by solvothermal synthesis from $\mathrm{Fe}^{\mathrm{III}}$ salts and organic acids, as reagents. In both types of nanoparticles, in addition to the iron oxides, the presence of coating of the NPs by the organic reagent was also detected. Finally, both samples were structural and magnetically characterized.

\section{EXPERIMENTAL}

All starting materials were commercially available reagents with analytical grade, which were used without further purification. The iron oxides $(\mathrm{FeO}-$ 1) and (FeO-2) were obtained by solvothermal synthesis in DMF in a $23 \mathrm{~mL}$ Teflon-lined stainless steel autoclave heated at $170{ }^{\circ} \mathrm{C}$ for $24 \mathrm{~h}$ under selfgenerated pressure. After slow cooling $\left(0.05^{\circ} \mathrm{C} / \mathrm{min}\right)$ at room temperature the solid product was filtered off and dried at $40{ }^{\circ} \mathrm{C}$. The reagents used to obtain $\mathrm{FeO}-1$ were 2-amino-3-hydroxypropanoic acid (1-serine), $\mathrm{FeCl}_{3} \cdot 4 \mathrm{H}_{2} \mathrm{O}$ and $\mathrm{Na}_{3} \mathrm{PO}$ in a molar ratio of $1: 2: 0.65$. FeO-2 was prepared using ascorbic acid, $\mathrm{FeCl}_{3} \cdot 4 \mathrm{H}_{2} \mathrm{O}$, and $\mathrm{K}_{2} \mathrm{CO}_{3}$, in a molar ratio of 1: 2: 0.65 . In both cases $5 \mathrm{~mL}$ of DMF were used as reaction solvent. Energy dispersive X-ray spectroscopy (EDXS) analyses performed on microcrystalline samples of $\mathrm{FeO}-1$ and $\mathrm{FeO}-2$, showed the presence of some type of iron oxide. Furthermore, both samples were analyzed by elemental analysis of carbon, obtaining a value of 1.1 and 6.0 $\%$ for $\mathrm{FeO}-1$ and $\mathrm{FeO}-2$ respectively.

X-Ray Powder Diffraction. Compounds FeO-1 and FeO-2 were analyzed by powder X-ray diffraction using a Siemens D5000 equipment, with $\mathrm{Cu}$ $K \alpha$, radiation and Bragg-Brentano geometry in the $5^{\circ} \leq 2 q \geq 80^{\circ}$ range. The analyses were performed using directly the microcrystalline samples. Electron Microscopy and X-ray Spectroscopy. Transmission electron microscopy (TEM) was done using a JEOL 2010 to determine the morphology, crystal structure and size distribution of the iron oxide nanoparticles. In order to make accurate size measurement, the magnifications were calibrated using SiC lattice fringes [10]. FTIR and DRIFT Spectroscopy. Absorption infrared and diffuse reflectance infrared spectra were recorded at room temperature on a Perkin Elmer Spectrum BX spectrophotometer. The infrared spectra were recorded 
in the $4000-400 \mathrm{~cm}^{-1}$ range using $\mathrm{KBr}$ pellets. DRIFT spectra were obtained between $4000-400 \mathrm{~cm}^{-1}$ on solid samples, in order to determine the presence of organic coating on the iron oxide compounds.

Magnetic Characterization. The magnetization curves of the synthesized iron oxide nanoparticles were recorded at room temperature on a vibrating sample magnetometer (VSM).

\section{RESULTS AND DISCUSSION}

\section{Synthesis and X-Ray Powder Diffraction}

The X-ray powder diffraction data of $\mathrm{FeO}-1$ presents peaks at $2 \theta$ of 32.8 , $35.6,40.5,49.3,54.0,62.4$ and $63.7^{\circ}$ (Figure 1a). These diffraction peaks with low intensity can be indexed with the planes (104), (110), (113), (024), (116), (214) and (300), respectively, corresponding to the rhombohedral phase of hematite $\left(\alpha-\mathrm{Fe}_{2} \mathrm{O}_{3}\right)$ [11]. Hematite is characterized by having a rhombohedrally centered hexagonal structure of the corundum type, with a close-packed oxygen lattice in which two-thirds of the octahedral sites are occupied by $\mathrm{Fe}^{\mathrm{III}}$ ions. The space group is $R \overline{3} c$ and the lattice parameters are $a=5.0356 \AA, c=$ 13.7489 ^ (JCPDS No. 79-0007) [12].

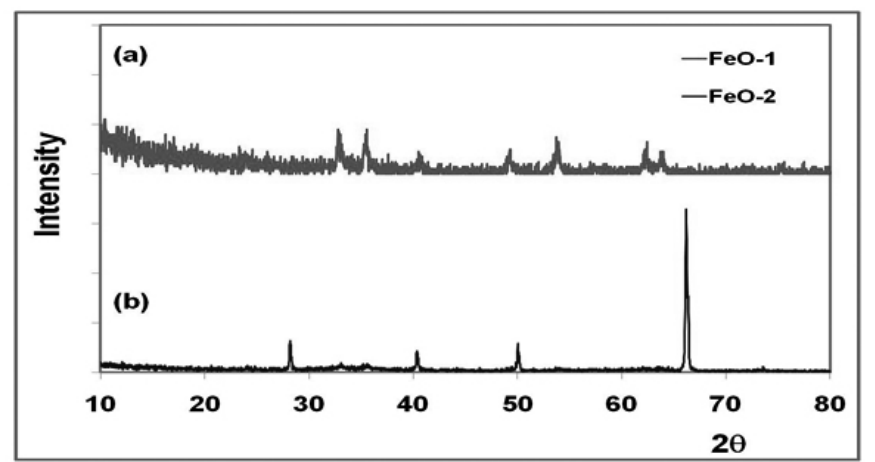

Figure 1. (a) Powder X-ray diffraction pattern of FeO-1. (b) Powder $\mathrm{X}$-ray diffraction pattern of $\mathrm{FeO}-2$.

The X-ray powder diffraction data obtained for $\mathrm{FeO}-2$ permit to identify the presence of at least two components. One of them, presents peak at 32.6 and $35.5^{\circ}$ which can be related with the (104) and (110) planes of $\alpha-\mathrm{F}_{2} \mathrm{O}_{3}$ (Figure 1b). The very low intensity of the peaks can be due to the low crystallinity of the synthesized hematite ( $\mathrm{FeO}-2)$. On the other hand, well-defined peaks at $2 \theta$ of $28.2,39.9,49.5$, and $66.3^{\circ}$ can be related to the presence of other crystalline iron oxides, such as maghemite $\left(\gamma-\mathrm{Fe}_{2} \mathrm{O}_{3}\right)$ or magnetite $\left(\mathrm{Fe}_{3} \mathrm{O}_{4}\right)$. Since both maghemite and magnetite have cubic spinel structure, the XRD patterns of these iron oxides are very much alike, and then the differentiation of these two phases using XRD is not straightforward [13].

$\gamma-\mathrm{Fe}_{2} \mathrm{O}_{3}$ is an allotropic form of $\mathrm{Fe}_{3} \mathrm{O}_{4}$, that is, these two iron oxides are crystallographic isomorphous. The main difference between these iron oxides is the presence of $\mathrm{Fe}^{\mathrm{II}}$ and $\mathrm{Fe}^{\mathrm{III}}$ ions in $\mathrm{Fe}_{3} \mathrm{O}_{4}$, while only $\mathrm{Fe}^{\mathrm{III}}$ ions exist in $\gamma$ - $\mathrm{Fe}_{2} \mathrm{O}_{3}$. Magnetite has the ferric ions distributed equally between the tetrahedral and octahedral sites, whose unit cell can be represented as $\left(\mathrm{Fe}^{3+}\right)_{8}\left[\mathrm{Fe}^{2.5+}\right]_{16} \mathrm{O}_{32}$, while maghemite as $\left(\mathrm{Fe}^{3+}\right)_{8}\left[\mathrm{Fe}^{3+}{ }_{5 / 6}-1 / 6\right]_{16} \mathrm{O}_{32}$, where the brackets () and [] designate tetrahedral and octahedral sites, respectively $[13 \mathrm{c}, \mathrm{d}]$. The X-ray diffraction pattern of maghemite possesses additional weak lines, which are attributed to vacancy ordering on the octahedral site. However, due to the lower intensities of these additional peaks, they are not used to differentiate magnetite from maghemite [13].

The presence of hematite as final product in $\mathrm{FeO}-1$ and $\mathrm{FeO}-2$ solids is not unusual if it is considered that hematite is the thermally most stable polymorph of all iron ${ }^{\text {III }}$ oxides, which can be obtained by the thermal conversion of a wide variety of iron ${ }^{\mathrm{II}}$ and iron ${ }^{\mathrm{III}}$ compounds. For example, it can be prepared from ferric salts in strongly acidic media, or by decomposition of iron chelates in alkaline media [12].

To measure the particle size, the first approach used was the Scherrer formula (equation 1):

$$
D=\frac{0.9 \lambda}{\beta \cos \theta}
$$

where, $D$ corresponds to the mean size, $\lambda$ is the $\mathrm{X}$-ray wavelength, $\beta$ is the line broadening at half the maximum intensity and $\theta$ is the Bragg angle. The accuracy of the mean size value obtained by this method is dependent of the peak width. Therefore, a good resolution of the X-ray powder diffractogram is significant to obtain the particle size. Consequently, the shape of the crystal and size distribution, and/or the Debye thermal broadening due to vibrations of the lattice, also strongly affects the precision of the size particle values [14].

Based on the (104) peak of FeO-1 and taking into account the poor resolution of the diffractogram, the mean size obtained from equation (1) was $13.1 \mathrm{~nm}$. This value is close to the value found by electron microscopy. In the case of $\mathrm{FeO}-2$, the diffractogram presents a very good resolution for the peaks at $28.2,39.9$ and $49.5^{\circ}$, being the most intense peak the one at $28.2^{\circ}$. Using this peak to estimate the size of the particle, the obtained value for the size is $75.7 \mathrm{~nm}$.

\section{Infrared and Diffuse Reflectance Infrared Spectroscopy}

The infrared spectrum obtained for the solid sample of FeO-1 shows absorptions at $3400,1615,540$ and $460 \mathrm{~cm}^{-1}$. Very weak absorptions are also observed at 1368 and $1054 \mathrm{~cm}^{-1}$. A similar band pattern was obtained for FeO-2 showing weak absorptions at 3380, 1604, 1354, 1052 and $580 \mathrm{~cm}^{-1}$ with shoulders at and 684 and $460 \mathrm{~cm}^{-1}$ (see Figure 2). For both samples the absorption in the low energy region $\left(<600 \mathrm{~cm}^{-1}\right)$ corresponds to $\mathrm{Fe}-\mathrm{O}$ vibrations, and the high energy region should be associated to the presence of organic species and water molecules. It is important to note that a broadening of the bands at low wave number is observed for $\mathrm{FeO}-2$. Literature data inform two absorptions for hematite at 440 and $526 \mathrm{~cm}^{-1}$, corresponding to the $E_{\mathrm{u}}$ and $A_{2 \mathrm{u}}+E_{\mathrm{u}}$ vibration modes respectively [15]. However, Sreeram et al. have assigned the absorptions at 471 and $587 \mathrm{~cm}^{-1}$ as characteristic of the $\mathrm{Fe}-\mathrm{O}$ stretching vibrations of crystalline hematite [9c]. Besides, similar absorptions near to 440 and $550 \mathrm{~cm}^{-1}$ have been assigned to the $T_{1}$ vibration modes of maghemite and magnetite with spinel crystal structure [15].
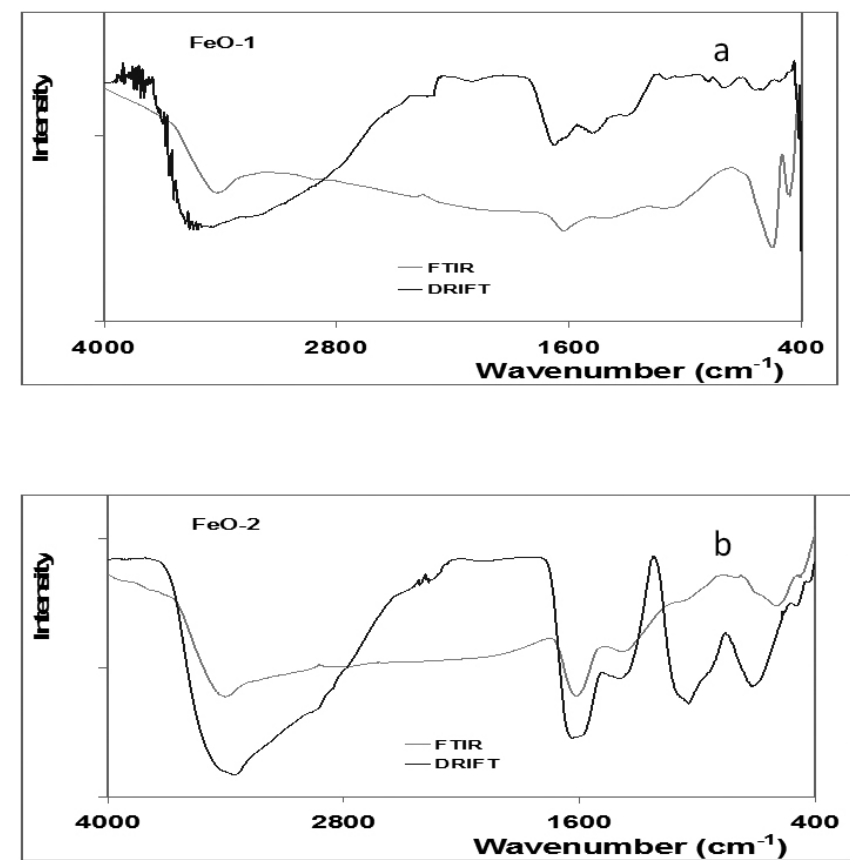

Figure 2. (a) Infrared (red line) and DRIFT (blue line) spectra of FeO-1. (b) Infrared (red line) and DRIFT (blue line) spectra of FeO-2.

According to literature data, the absorptions observed between 460 and 540 $\mathrm{cm}^{-1}$ in $\mathrm{FeO}-1$, can be related with the $E_{u}$ and $A_{2}+E_{u}$ hematite vibration modes, respectively. In the case of $\mathrm{FeO}-2$, the broadening of the band centered at 580 $\mathrm{cm}^{-1} \mathrm{can}$ be attributed to the mixture of iron oxides present in this sample. This absorption, together with the shoulder observed at $460 \mathrm{~cm}^{-1}$ should be related with the characteristic $T_{1 \mathrm{u}}$ vibration modes of spinel type iron oxides, such as maghemite and magnetite.

On the other hand, the bands around $1100 \mathrm{~cm}^{-1}$ can be assigned to the symmetric stretching vibration of the $\mathrm{C}-\mathrm{O}$ bond, which is present in both 
organic molecules (serine and ascorbic acid) used as reagents to obtain the FeO-1 and FeO-2 iron oxide particles [9c]. The absorptions at 3500 and 1650 $\mathrm{cm}^{-1}$ are attributed to the water molecules adsorbed on the surface of FeO-1 and $\mathrm{FeO}-2$ [16].

The DRIFT spectra of FeO-1 and FeO-2 are also given in figure 2. The presence of organic species, together to the synthesized iron oxide nanoparticles, is also evidenced by the absorption pattern obtained for both samples. Wide absorptions at approximately 3400,1640,1450, 1100 and $750 \mathrm{~cm}^{-1}$ are observed. The bands at 1660 and $1450 \mathrm{~cm}^{-1}$ can be assigned to carboxylate groups [17].

The use of diffuse reflectance spectroscopy has allowed to identify the presence of organic compounds in the samples, which correspond to serine in the case of compound FeO-1, and ascorbic acid in the case of FeO-2. Therefore, the presence of these organic absorptions is strong evidence that the organic compounds are covering the iron oxide nanoparticle surface.

\section{Electron Microscopy}

The morphology and size of the particles were analyzed by electron microscopy techniques. Representative SEM and TEM images of $\mathrm{FeO}-1$ at different magnification are shown in Figure 3. Low magnification SEM image (see Figure 3a) shows circular plates of uniform size sorted in different directions, giving morphologies similar to flowers. This arrangement is lost when the SEM image is obtained at a higher magnification (see Figure 3b).
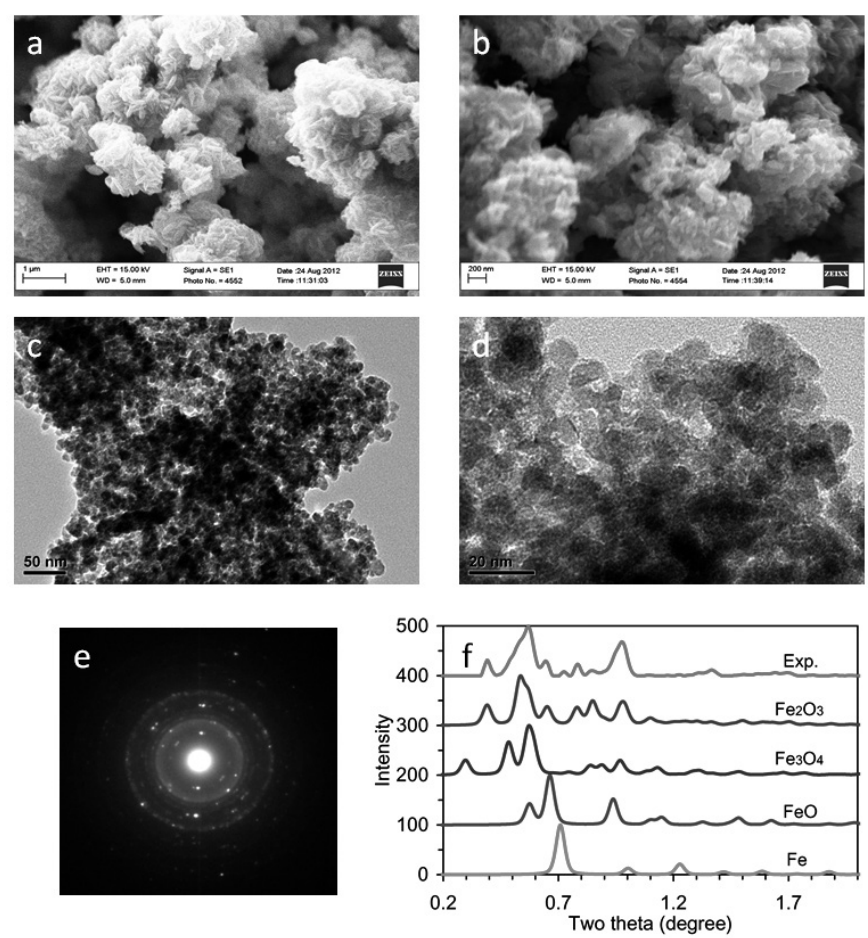

Figure 3. SEM and TEM images of FeO-1. (a) Overview SEM image in a low magnification; (b) Enlarged SEM image; (c) Overview TEM image in a low magnification; (d) Enlarged TEM image; (e) Electron diffraction pattern showing poly rings from iron oxide nanoparticles; (f) Electron diffraction pattern of different iron oxide samples.

At this resolution the solid (FeO-1) does not show a defined morphology. Higher magnifications were obtained through of TEM analysis. A representative TEM image of FeO-1 is shown in Figure 3c, which is magnified in Figure 3d. It is seen that these NPs have uniform and small size. The presence of nanocrystals of iron oxide nanoparticles in the sample is evidenced by the electron diffraction pattern in Figure 3(e), showing the existing high crystallinity. The intensity profile of the electron diffraction pattern is retrieved after removing the background in the way reported previously [18], as shown in Figure 3(f). As compared with the simulations using structural models of $\mathrm{Fe}_{2} \mathrm{O}_{3}, \mathrm{Fe}_{3} \mathrm{O}_{4}, \mathrm{FeO}$ and $\mathrm{Fe}$, the experimental data are very consistent with the $\mathrm{Fe}_{2} \mathrm{O}_{3}$ structure. The size distribution given in Figure 4 corresponds to a size variation between 1.8 and $20 \mathrm{~nm}$, with a mean value of $10.1 \mathrm{~nm}$, and standard deviation of $3.2 \mathrm{~nm}$, based on the measurement of 219 nanoparticles. The nanoparticle size is found to be in a narrow size range [19]. It is important to remark that the mean size obtained by the Scherrer formula $(13.1 \mathrm{~nm})$ is very close to that estimated by TEM microscopy $(10.1 \mathrm{~nm})$.

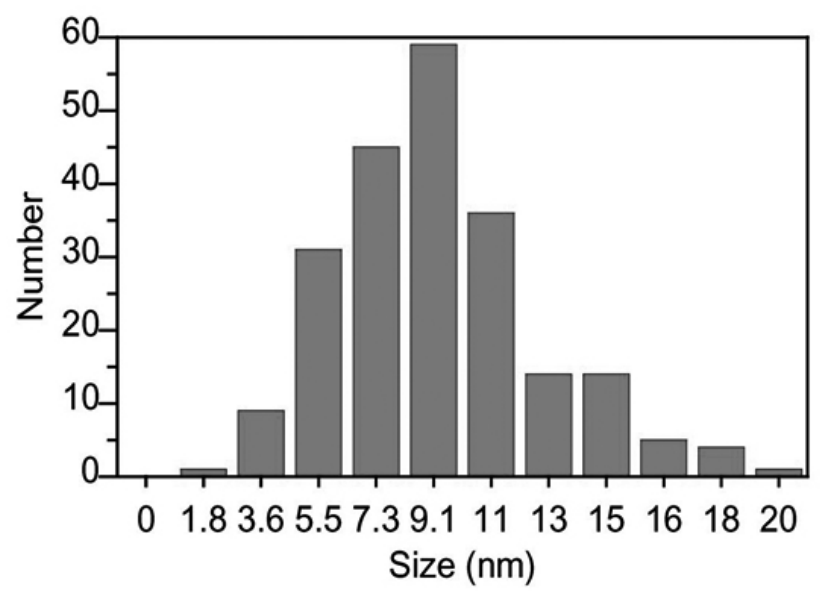

Figure 4. Size distribution of iron oxide nanoparticles in sample FeO-1.

The SEM and TEM images obtained for FeO-2 are given in Figure 5. Unlike the NPs of iron oxide of FeO-1, the SEM images obtained for $\mathrm{FeO}-2$ (see Figure $5 \mathrm{a}$ and $5 \mathrm{~b}$ ) do not have small and uniform size, and also do not present a special morphology. Some agglomerates lower than $200 \mathrm{~nm}$ can be evidenced from the SEM image given in Figure 5b. It was impossible to determine the size distribution from the TEM image obtained for FeO-2 (see Figure $5 \mathrm{c}$ ), because its low quality. Furthermore, a very low crystallinity of the sample of $\mathrm{FeO}-2$ as compared with that of $\mathrm{FeO}-1$ can be observed by the electron diffraction pattern given in Figure 5(d). Therefore, from the SEM and TEM data of FeO-2, it is only possible to infer that the average size of the NPs is significantly larger than that in FeO-1, which is consistent with the XRD results.
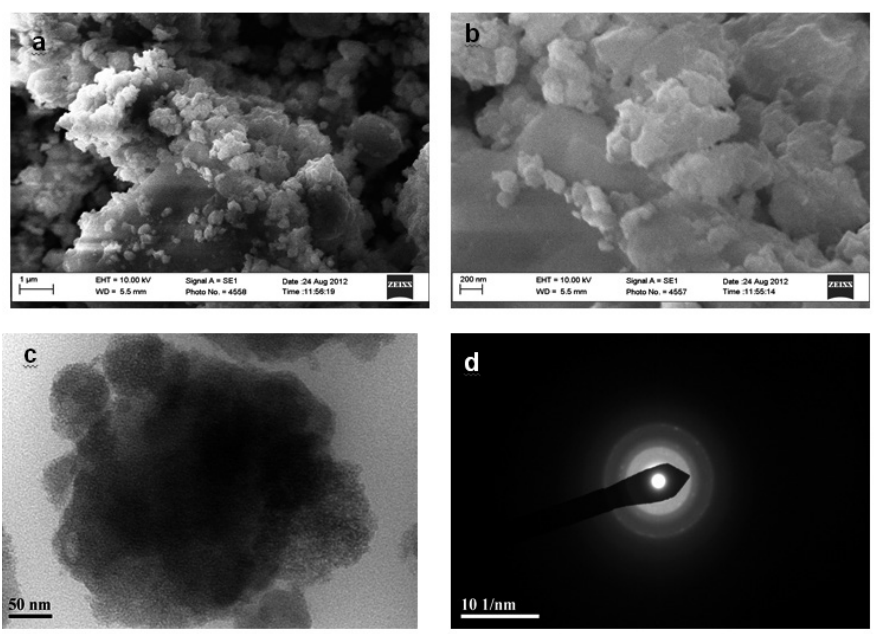

Figure 5. SEM and TEM images of FeO-2. (a) Overview SEM image in a low magnification; (b) Enlarged SEM image; (c) Overview TEM image in a low magnification; (e) Electron diffraction pattern of FeO-2 sample.

\section{Magnetic Measurements}

The magnetization curves of the synthesized iron oxide nanoparticles were measured at room temperature, under a maximum applied field of $10 \mathrm{kOe}$ Hysteresis loops $M(H)$ of FeO-1 and FeO-2 are shown in Figure 6. 

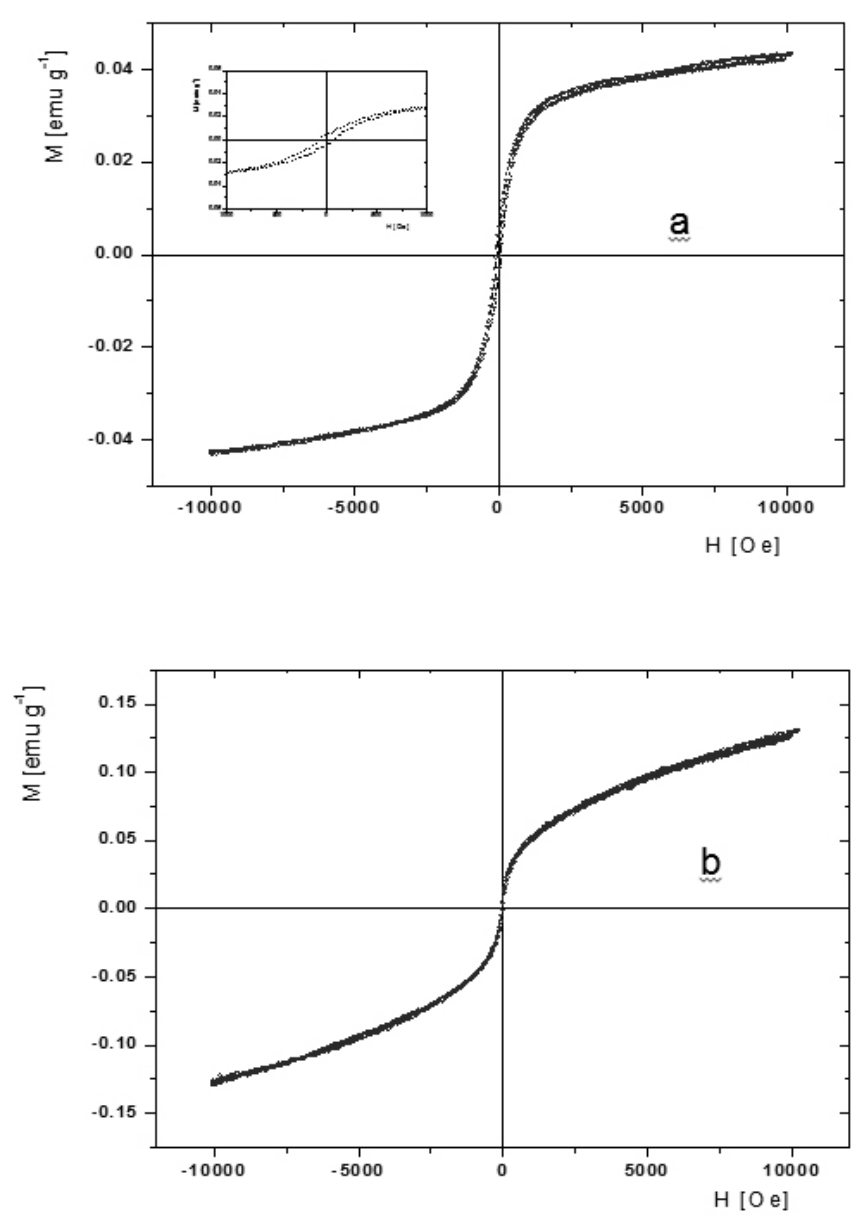

Figure 6. (a) Hysteresis loop of FeO-1. (b) Hysteresis loop of FeO-2.

Although a complete magnetic saturation is not achieved in either cases, the $M(H)$ plot obtained for FeO-1 shows that the magnetization of the sample is closer to the saturation value than that for FeO-2. For FeO-1, a fast increase of the magnetization is observed until $2.5 \mathrm{kOe}$ and at higher field, the near saturation value obtained for $\mathrm{FeO}-1$ was $0.04 \mathrm{emu} \mathrm{g}^{-1}$. Hysteresis is observed for $\mathrm{FeO}-1$, indicating the ferromagnetic behavior at room temperature. From the $M(H)$ plot it is possible to obtain values of coercivity $\left(H_{\mathrm{c}}\right)$ and a remanent magnetization $\left(M_{\mathrm{r}}\right)$ of $53 \mathrm{Oe}$ and $0.005 \mathrm{emu} \mathrm{g}^{-1}$ respectively (inset Figure 6a). These values are in the order of the reported ones for analogous flower-like $\alpha-\mathrm{Fe}_{2} \mathrm{O}_{3}[20]$.

The bulk hematite is classified as a weak ferromagnetic material at room temperature, presenting a weak magnetization. It is known that the particles of a ferromagnetic material change from a multidomain to a single domain, when the size of the magnetic particles decreases, thus causing the phenomenon of superparamagnetism [21]. Considering previous reports, the hematite critical size for the transition from ferromagnetic to superparamagnetic behavior, depends on the particles shape, the strain and defects in the particles induced by the different synthetic routes [22-24]. Thus, for spherical hematite, the reported biggest threshold diameter is about $41 \mathrm{~nm}$, below this size the particles become superparamagnetic $(\mathrm{Hc} \approx 0)$ [25]. Nonetheless, for one dimensional nanostructures (hematite nanorods), the value is $15 \sim 25 \mathrm{~nm}$ in breadth and $170 \sim 330 \mathrm{~nm}$ in length. On the other hand, for larger particles of hematite the coercivity depends mainly on the grain size and the morphology. Svoboda et al. noted that depending on the microstructure of the hematite particles, the coercivity $\mathrm{H}_{\mathrm{c}}$ may range from 0.3 to $3 \mathrm{kOe}$ [26]. In the case of FeO-1, the coercivity value is much lower than the range reported by Svoboda. Therefore, supposing a multidomain structure for the synthesized hematite nanoparticles, which could explain the observed coercivity, does not appear as coherent if the average size of $10 \mathrm{~nm}$ is considered. Therefore, the fact that a non-complete magnetic saturation was observed at the maximum applied magnetic field, can be explained by the increase of the surface spin disorder, and the very small coercivity can be attributed to some magnetically blocked spins at room temperature [27].

The hysteresis loop for FeO-2 shows that the remanence and coercivity are very much lower than that observed for FeO-1 $\left(M_{r}=0.004\right.$ emug $^{-1}$ and $H=10$ Oe). Even though the magnetization values are higher than those obtained for FeO-1 $\left(M_{s}=0.13 \mathrm{emu} \mathrm{g}^{-1}\right)$. Taking into account that bulk maghemite and magnetite have a magnetic saturation of 80 and $92 \mathrm{emu} \mathrm{g}^{-1}$ at room temperature, respectively, and considering the $M_{s}$ value obtained for $\mathrm{FeO}-$ 2 , it can be concluded that this synthesized iron oxide should be constituted mainly by $\alpha-\mathrm{Fe}_{2} \mathrm{O}_{3}$. According with the complete experimental evidence of these synthesized IONPs we can conclude that the main constituent in both samples is hematite $\left(\alpha-\mathrm{Fe}_{2} \mathrm{O}_{3}\right)$, while the sample FeO-2 also could contain a minor amount of maghemite or magnetite. However, due to structural similarities between maghemite and magnetite and also by their spectroscopic and magnetic properties, the identification of the constituents in sample FeO-2 remains a challenge.

From a chemical point of view, the process for the preparation of some iron oxides starting from iron oxyhydroxide, which can be obtained from iron (III) salts is presented in the following scheme [13a,28]:

$$
\begin{array}{rl}
\mathrm{FeX}_{3}+3 \mathrm{H}_{2} \mathrm{O}+3 \mathrm{RNH}_{2} & \longrightarrow \mathrm{Fe}(\mathrm{OH})_{3}+3 \mathrm{RNH}_{3} \mathrm{X} \\
\mathrm{Fe}(\mathrm{OH})_{3} & \mathrm{FeOOH} \\
\mathrm{FeOOH} \stackrel{\text { dehydratation }}{\longrightarrow} \alpha-\mathrm{Fe}_{2} \mathrm{O}_{3} \stackrel{\text { reduction }}{\longrightarrow} \mathrm{Fe}_{3} \mathrm{O}_{4} \stackrel{\text { oxidation }}{\longrightarrow} \gamma-\mathrm{Fe}_{2} \mathrm{O}_{3}
\end{array}
$$

In this chemical process, the magnetite appears as the first product obtained from hematite. However, in the solvothermal synthesis, redox processes can easily occur. Taking into account that hematite and maghemite correspond to the most frequent mixture of iron oxides present in nature, it is possible to infer that sample $\mathrm{FeO}-2$ is composed of hematite and maghemite.

\section{CONCLUSIONS}

Synthesized hematite and a mixture of hematite and maghemite nanoparticles were obtained under solvothermal conditions. Both samples were characterized by powder X-ray diffraction, electronic microscopy and magnetic measurements. In both iron oxide samples the presence of an organic coating can be evidenced by diffuse reflectance FTIR spectroscopy. The magnetic characterization of the iron oxide nanoparticles shows that the hematite sample, $\mathrm{FeO}-1$, presents a weak ferromagnetic behavior with a very low coercivity value. This is greater than the observed for the synthesized hematite-maghemite mixture, $\mathrm{FeO}-2$. In both cases complete magnetization saturation is not achieved, indicating the increasing surface spin disorder in both synthesized iron oxides samples. Furthermore, the magnetization values obtained for $\mathrm{FeO}-2$, suggests that the synthesized hematite is the main component in the sample.

\section{ACKNOWLEDGMENTS}

The authors acknowledge financial support from FONDECYT 1090477, DI-UNAB-104-12/R and Proyecto Basal CEDENNA, Financiamiento Basal FB0807.

\section{REFERENCES}

1. (a) L. LaConte, N. Nitin, G. Bao. Mater. Today. 8, 32, (2005). (b) V. Balzani. Small. 1, 278, (2005). (c) G Cárdenas-Triviño, O. GodoyGuzmán, G. Conterreas. J. Chil. Chem. Soc. 54, 6, (2009). (d) Y. León, I. Brito, G. Cárdenas, O. Godoy. J. Chil. Chem. Soc. 54, 51, (2009).

2. (a) W. Wu, Q. He, Ch. Jiang. Nanoscale Res. Lett. 3, 397, (2008). (b) S. Laurent, D. Forge, M. Port, A. Roch, C. Robic, L. V. Elst, R.N. Muller. Chem. Rev. 108, 2064, (2008).

3. (a) L.A. Thomas, L. Dekker, M. Kallumadil, P. Southern, M. Wilson, S.P. Nair, Q.A. Pankhurst, I.P. Parkin. J. Mater. Chem. 19, 6529, (2009). (b) J.S. Jiang, Z.F. Gan, Y. Yang, B. Du, M. Qian, P. Zhang. J. Nanopart. Res. 11, 1321, (2009). (c) K. Chen, J. Xie, H. Xu, D. Behera, M.H. Michalski, S. Biswal, A. Wang, X. Chen. Biomaterials. 30, 6912, (2009). (d) C. Altavilla, E. Ciliberto Inorganic Nanoparticles. Synthesis, Applications, and Perspectives. CRC Press, Taylor and Francis Group, USA, 2010. (e) A.S. Teja, P.-Y. Koh. Prog. Cryst. Growth Ch. 55, 22, (2009). (f) Q. Liu, Z.M. Cui, Z. Ma, S.W. Bian, W.G. Song, L.J. Wan. Nanotechnology. 18, 385605, (2007). (g) F. Shi, M.K. Tse, M.M. Pohl, A. 
Brückner, S. Zhang, M. Beller, Angew. Chem. Int. Edit. 46, 8866, (2007). (h) S. Al-Sayari, A.F. Carley, S.H. Taylor, G.J. Hutchings. Top. Catal. 44, 123, (2007). (i) A.K. Kandalam, B. Chatterjee, S.N. Khanna, B.K. Rao, P. Jena, B.V. Reddy. Surf. Sci. 601, 4873, (2007). (j) H. Gaedcke in Industrial Inorganic Pigments, G. Buxbaum ed. Wiley-VCH, Weinheim, 1998; pp. 181-188. (k) U.T. Lam, R. Mammucari, K. Suzuki, N.R. Foster. Ind. Eng. Chem. Res. 47, 599, (2008). (1) R.M. Cornell, U. Schwertmann The Iron Oxides. Structure, Properties, Reactions, Occurrences and Uses, Wiley-VCH, Weinheim, 2003. (m) D. Ruiz, C. Mella, J.L.G. Fierro, P. Reyes. J. Chil. Chem. Soc. 57, 1394, (2012).

4. N. Sounderya, Y. Zhang. Recent Patents on Biomedical Engineering 1, 34, (2008).

5. (a) A. Figuerola, R. Di Corato, L. Manna, T. Pellegrino. Pharmacol. Res. 62, 126, (2010). (b) J. Chomoucka, J. Drbohlavova, D. Huska, V. Adam, R. Kizek, J. Hubalek. Pharmacol. Res. 62, 144, (2010). (c) A. K. Gupta, M. Gupta. Biomaterials. 26, 3995, (2005). (d) P. Haddad, T.M. Martins, L. D Souza-Li, L.M. Li, K. Metze, R.L. Adam, M. Knobel, D. Zanchet. Mater. Sci. Eng. C. 28, 489, (2008).

6. (a) A. Millan, F. Palacio, A. Falqui, E. Snoeck, V. Serin, A. Bhattacharjee, V. Ksenofontov, P. Gutlich, I. Gilbert. Acta Mater. 55, 2201, (2007). (b) G. Zhang, Y. Liao, I. Baker. Mater. Sci. Eng. C-Mater Biol Appl. 30, 92, (2010). (c) J. Gao, H. Gu, B. Xu. Acc. Chem. Res. 42, 1097, (2009). (d) N. L. Rosi, Ch. A. Mirkin. Chem. Rev. 105, 1547, (2005). (e) R. Qiao, Ch. Yang, M. Gao. J. Mater. Chem. 19, 6274, (2009).

7. (a) J. T. Nurmi, P.G. Tratnyek, V. Sarathy, D. R. Baer, J.E. Amonette, K. Pecher, Ch. Wang, J.C. Linehan, D.W. Matson, R.L. Penn, M. D. Driessen. Environ. Sci. Technol. 39, 1221, (2005). (b) H. Karami. J. Clust. Sci. 21, 11, (2010). (c) G. Zhen, B.W. Muir, B.A. Moffat, P. Harbour, K.S. Murray, B. Moubaraki, K. Suzuki, I. Madsen, N. AgronOlshina, L.Waddington, P. Mulvaney, P.G. Hartley. J. Phys. Chem. C. 115, 327, (2011). (d) C. Han, J. Xie, C. Deng, D. Zhao. J. Chil. Chem. Soc. 57, 1372, (2012).

8. (a) J.P. Ge, Y.X. Hu, M. Biasini, C.L. Dong, J.H. Guo, W.P. Beyermann, Y.D. Yin. Chem. Eur. J. 13, 7153, (2007). (b) T. Hyeon. Chem. Commun. 927, (2003). (c) Y. Wenguang, Z. Tonglai, Z. Jianguo, G. Jinyu, W. Ruifeng. Prog. Chem. 19, 884, (2007). (d) Y.Y. Sun, G.Z. Guo, B.H. Yang, W. Cai, Y. Tian, M.H. He, Y.Q. Liu. Physica B. 406, 1013, (2011).

9. (a) A.M. Schrand, M.F. Rahman,.S.M. Hussain, J.J. Schlager, D.A.Smith, A.F. Syed. WIREs Nanomed. Nanobiotechnol. 2, 544, (2010). (b) D.A. Canelas, K.P. Herlihy, J.M. DeSimone. WIREs Nanomed. Nanobiotechnol. 1, 391, (2009). (c) M. Nidhin, R. Indumathy, K.J. Sreeram, B.U. Nair. Bull. Mater. Sci. 31, 93, (2008). (d) K.J. Sreeram, M. Nidhin, B.U. Nair. Colloid Surface B. 71, 260, (2009). (e) M.P.S. de
Almeida, K.L. Caiado, P.P.C. Sartoratto, D.O. Cintra, E. Silva , A.R. Pereira, P.C. Morais. J. Alloy. Compd. 500, 149, (2010).

10. Z.P. Luo. Acta Mater. 54, 47, (2006).

11. R.L. Blake, R.E, Hessevick, T. Zoltai, L.W. Finger. Am. Mineral. 51, 123, (1966).

12. (a) H. Cao, G. Wang, L. Zhang, Y. Liang, S. Zhang, X. Zhang. Chem. Phys. 7, 1897, (2006). (b) R.K Zboril, M. Mashlan, D. Petridis. Chem. Mater. 14, 969, (2002).

13. (a) M. Gotic, G. Košcec, S. Music. J. Mol. Struct. 924-926, 347, (2009) (b) X. Wang, X. Chen, X. Ma, H. Zheng, M. Ji,, Z. Zhang. Chem. Phys. Lett. 384, 391, (2004). (c) R. Grau-Crespo, A.Y. Al-Baitai, I. Saadoune, N.H. De Leeuw. J. Phys-Condens. Mat. 22, 255401, (2010). (d) K. Haneda, A. H. Morrish. J. Phys. Colloques. 38, Cl-321, (1977).

14. V. Ciupină, S. Zamfirescu and G. Prodan in Evaluation of Mean Diameter values using Scherrer Equation Applied to Electron Diffraction Images, Nanotechnology- Toxicological Issues and Environmental Safety, P.P. Simeonova, N. Opopol, M.I. Luster eds. Springer, Netherlands, 2007; pp. 231-237.

15. (a) A.M. Jubb, H.C. Allen. ACS Appl. Mater. Interfaces. 2, 2804, (2010) (b) S. Onari, T. Arai, K. Kudo. Phys. Rev. B. 16, 1717, (1977). (c) C. J. Serna, J. L. Rendon, J.E. Iglesias. Spectrochim. Acta, A. 38, 797, (1982).

16. R. Arbain, M. Othman, S. Palaniandy. Miner. Eng. 24, 1, (2011).

17. I. F. Trotter, H.W. Thompson, F. Wokes. Biochem. J. 42, 601, (1948).

18. Z.P. Luo, Y. Vasquez, J.F. Bondi, R.E. Schaak. Ultramicroscopy 111, 1295, (2011).

19. Z.P. Luo. J. Mater. Sci. 45, 3228, (2010).

20. S. Zeng, K. Tang, T. Li, Z. Liang, D. Wang, Y. Wang, Y. Qi, W. Zhou. J. Phys. Chem. C. 112, 4836, (2008).

21. N. Amin, S. Arajs and E. Matijevic, Phys. Status Solidi A, 104, K65, (1987).

22. T. P. Raming, A. J. A. Winnubst, C. M. van Kats, A. P. Philipse, $J$. Colloid Interf. Sci. 249, 346, (2002).

23. F. Bodker, M. F. Hansen, C. B. Koch, K. Lefmann, S. Morup. Phys. Rev. B. 61, 6826, (2000).

24. B. Tang, G. Wang, L. Zhuo, J. Ge, L. Cui. Inorg. Chem. 45, 5196, (2006).

25. Z. Gan, A. Zhao, Q. Gao, M. Zhang, D. Wang, H. Guo,W. Tao, D. Li, E. Liu, R. Mao. RSC Adv. 2, 8681, (2012).

26. J. Svoboda Magnetic Techniques for the Treatment of Materials, Springer, USA, 2004.

27. P.G. Bercoff, H.R. Bertorello. Appl. Phys. A-Mater. 100, 1019, (2010).

28. Z. Li, X. Lai, H. Wang, D. Mao, C. Xing, D. Wang. Nanotechnology. 20, 245603, (2009). 